OPEN ACCESS

Edited by:

Kimberly Huey,

Drake University, United States

Reviewed by:

Robin Souron,

Laboratoire Interuniversitaire de Biologie de la Motricité (LIBM),

France

Kyle T. Ebersole,

University of Wisconsin-Milwaukee,

United States

*Correspondence:

Alberto Rainoldi

alberto.rainoldi@unito.it

Specialty section:

This article was submitted to

Exercise Physiology,

a section of the journal

Frontiers in Physiology

Received: 20 August 2018 Accepted: 05 October 2018 Published: 24 October 2018

Citation:

Boccia G, Dardanello D, Brustio $P R$, Tarperi $C$, Festa $L$, Zoppirolli C, Pellegrini B, Schena F and Rainoldi A (2018) Neuromuscular

Fatigue Does Not Impair the Rate of Force Development in Ballistic

Contractions of Submaximal

Amplitudes. Front. Physiol. 9:1503.

doi: 10.3389/fphys.2018.01503

\section{Neuromuscular Fatigue Does Not Impair the Rate of Force Development in Ballistic Contractions of Submaximal Amplitudes}

\section{Gennaro Boccia ${ }^{1,2}$, Davide Dardanello', Paolo Riccardo Brustio', Cantor Tarperi ${ }^{3}$, Luca Festa ${ }^{3}$, Chiara Zoppirolli2,3, Barbara Pellegrini ${ }^{2,3}$, Federico Schena ${ }^{2,3}$ and Alberto Rainoldi ${ }^{*}$}

${ }^{1}$ NeuroMuscularFunction Research Group, School of Exercise \& Sport Sciences, Department of Medical Sciences, University of Turin, Turin, Italy, ${ }^{2}$ CeRiSM Research Center for Sport, Mountain, and Health, University of Verona, Rovereto, Italy, ${ }^{3}$ School of Sport and Exercise Sciences, Department of Neurosciences, Biomedicine and Movement Sciences, University of Verona, Verona, Italy

The effect of muscle fatigue on rate of force development (RFD) is usually assessed during tasks that require participants to reach as quickly as possible maximal or nearmaximal force. However, endurance sports require athletes to quickly produce force of submaximal, rather than maximal, amplitudes. Thus, this study investigated the effect of muscle fatigue induced by long-distance running on the capacity to quickly produce submaximal levels of force. Twenty-one male amateur runners were evaluated before and shortly after a half-marathon race. Knee extensors force was recorded under maximal voluntary and electrically evoked contractions. Moreover, a series of ballistic contractions at different submaximal amplitudes (from 20 to $100 \%$ of maximal voluntary force) was obtained, by asking the participants to reach submaximal forces as fast as possible. The RFD was calculated for each contraction. After the race, maximal voluntary activation, resting doublet twitch, maximal force, and RFD during maximal contraction decreased $(-12,-12,-21$, and $-19 \%$, respectively, all $P$-values $<0.0001)$. Nevertheless, the RFD values measured during ballistic contractions up to $60 \%$ of maximal force were unaffected (all $P$-values $>0.4$ ). Long-distance running impaired the capacity to quickly produce force in ballistic contractions of maximal, but not of submaximal, amplitudes. Overall, these findings suggest that central and peripheral fatigue do not affect the quickness to which muscle contracts across a wide range of submaximal forces. This is a relevant finding for running and other daily life activities that rely on the production of rapid submaximal contractions rather than maximal force levels.

Keywords: central fatigue, peripheral fatigue, explosive strength, rate of force development scaling factor, endurance running 


\section{INTRODUCTION}

Muscle fatigue can be defined as an exercise-induced decreased capacity to generate maximal force (Gandevia, 2001). Thus, measuring reductions in maximal voluntary contraction force (MVCF) is considered as the most valid and widespread approach to measure muscle fatigue (Millet and Lepers, 2004; Place et al., 2010). It is a sensitive and reliable procedure, which is easy to administer in a variety of research settings (Wilson and Murphy, 1996). Nevertheless, its functional value has been recently questioned (Maffiuletti et al., 2016; Rodriguez-Rosell et al., 2017). Indeed, in many daily and sport activities, the time required to develop maximal force (300 ms or more) is longer than the time available to develop force (Maffiuletti et al., 2016). Therefore, under the time-restricted conditions of short muscle actions, the ability to rapidly produce force is considered functionally more important than maximal force (Maffiuletti et al., 2016; Rodriguez-Rosell et al., 2017). Moreover, it was shown that the capacity to maintain a rapid force production is important in fatigued states, even though this aspect has received little attention in the literature so far (Girard et al., 2015; Morel et al., 2015).

The rate of force development (RFD) reflects the ability to rapidly increase muscle force after the onset of an explosive voluntary contraction (Maffiuletti et al., 2016; Rodriguez-Rosell et al., 2017). RFD has been usually assessed in contractions that require participants to reach as quickly as possible maximal or near-maximal force (usually higher than $80 \%$ of maximal force; Folland et al., 2014). Throughout the manuscript we will consider the RFD measured following the above-mentioned procedure as maximal RFD. Maximal RFD is likely to be an important determinant of performance in explosive tasks like the shot put (Zaras et al., 2016) or the vertical jump (McLellan et al., 2011). However, it may not reflect the demands of those sports and daily life activities that rely on submaximal muscle contractions, such as endurance running. Indeed, Kulmala et al. (2016) showed that when running at $4.1 \mathrm{~m} \cdot \mathrm{s}^{-1}$, the peak force exerted by knee extensors reached the $63 \pm 17 \%$ of their maximal force (calculated during hopping task). Thus, in endurance running knee extensors do not exert the maximal force they are capable of, rather they quickly produce a certain amount of force that is needed at each step for the propulsion of the body at a given speed (Kyrolainen et al., 2005; Kluitenberg et al., 2012; Bigouette et al., 2016). For this reason, we suggest that the RFD measured during ballistic contraction of submaximal amplitude could have higher ecological validity than maximal RFD in endurance activities, such as running. Consequently, a distinction should be drawn between ballistic contraction of maximal amplitudes, usually adopted in the literature to calculate the maximal RFD, and ballistic contraction of submaximal amplitudes, which are possibly more relevant for endurance running. While it has been demonstrated that running-based fatiguing protocols impair the maximal RFD (Oliveira et al., 2013; Boccia et al., 2017a, 2018), it is unknown to what extent muscle fatigue may impair the ability to quickly produce forces of submaximal amplitudes.

To this aim, a protocol usually adopted to calculate the so-called RFD scaling factor (RFD-SF) provides an appealing approach (Bellumori et al., 2011, 2013; Casartelli et al., 2014; Djordjevic and Uygur, 2017). The protocol consists in a series of quick and fast (i.e., ballistic) contractions performed with different submaximal amplitudes (namely from 20 to $100 \%$ of MVCF) (Freund and Budingen, 1978; Ghez and Vicario, 1978; Wierzbicka et al., 1991; Klass et al., 2008). In each ballistic contraction the individuals are asked to roughly reach a given submaximal force as fast as possible (the emphasis is on the quickness of the contraction rather than on the accurateness). The RFD measured in each contraction thus quantify the capacity to quickly produce submaximal force (Bellumori et al., 2011, 2013; Casartelli et al., 2014; Djordjevic and Uygur, 2017).

In the aforementioned protocol, the RFD-SF consists in the slope of the linear relationship between the peak force and the peak RFD obtained in each ballistic contraction (Bellumori et al., 2011, 2013; Casartelli et al., 2014; Djordjevic and Uygur, 2017). This relationship has been investigated since the late 1970 s (Freund and Budingen, 1978; Ghez and Vicario, 1978). The consistent finding since then was a linear increase in RFD at rising force amplitudes: i.e., the higher the force required, the quicker the contraction (Freund and Budingen, 1978; Ghez and Vicario, 1978; Wierzbicka et al., 1991; Van Cutsem et al., 1998). While the physiological mechanisms underpinning the RFD-SF are far from being elucidated, the available studies suggest that RFD-SF measurement may inform about the important features of movement initiation and quickness of force production (Wierzbicka et al., 1991; Bellumori et al., 2011). Indeed, the RFD-SF is known to be lower in older adults compared to young adults (Klass et al., 2008; Bellumori et al., 2013) and also in people with Parkinson's disease compared to healthy people (Wierzbicka et al., 1991). Furthermore, RFD-SF increases after a period of power training both in young and elderly people (Van Cutsem et al., 1998; Bellumori et al., 2017), its improvement being related to an increase in motor units discharge rate (Van Cutsem et al., 1998; Klass et al., 2008). The fact that RFD-SF can reflect neural factors was furthermore substantiated by the study of Van Cutsem and Duchateau (2005). They showed that performing ballistic contractions from a sustained contraction, compared to a resting state, decreased the instantaneous motor unit discharge rate and the RFD, thereby reducing the RFDSF (Van Cutsem and Duchateau, 2005). Beyond neural factors, biochemical and mechanical muscle properties such as muscle fiber type, fiber shortening velocity, and elastic properties of the muscle-tendon unit (Maffiuletti et al., 2016) are known to influence RFD, even though their effect on RFD-SF has not been investigated so far. However, despite the neuromuscular correlates are not clear, RFD-SF has been considered to be an appealing measure to compare individuals and populations independently to their strength (Bellumori et al., 2011), since it is not affected by maximal force capacity. It is well known that both central and peripheral mechanisms contribute to impair maximal RFD after fatiguing exercise (Buckthorpe et al., 2014; Boccia et al., 2016, 2018; Maffiuletti et al., 2016), but the impact of fatigue on RFD-SF has not been yet investigated.

Therefore, the aims of the study were to assess if muscle fatigue induced by long-distance running would impair (1) the RFD exerted in ballistic contractions of submaximal 
amplitudes; and/or (2) the RFD-SF of knee extensor muscles. We hypothesized that a half-marathon race decreases the RFD in ballistic contractions of submaximal amplitudes and the RFD-SF.

\section{MATERIALS AND METHODS}

\section{General Overview}

The study was performed during a scientific event called Run For Science, hosted by the University of Verona (Italy) in April 2017, for details see (Lippi and Schena, 2017). In this event, participants competed in an official half-marathon race certified by Italian Track and Field Federation. The day of the race the weather was sunny, with no wind, the air temperature was $18^{\circ}$ and humidity $70 \%$ and these conditions were quite stable along the duration of the event. The start waves were assigned to participants based on the individual estimated race time, to avoid many participants reaching simultaneously the testing station. The assessments consisted in a series of voluntary and electrically evoked contractions of the knee extensors. Participants were involved in two measurement sessions: the first was performed the day before the race (PRE), and the second shortly after the race (POST). A schematic representation of the experimental procedures is reported in the Figure 1. Participants were instructed to refrain from performing strenuous physical activity in the $24 \mathrm{~h}$ before the first experimental session. They were also asked to refrain from consuming caffeine in the $24 \mathrm{~h}$ before the first experimental session and before the race. During this first session, participants were familiarized with the maximal voluntary contractions, peripheral nerve electrical stimulation, and RFD-SF protocol. For that purpose, participants repeated two or three trials of the test procedures until they were able to produce consistent results. Before the neuromuscular testing at PRE, participants performed a warm-up consisting of $15 \mathrm{~min}$ of outdoor running at an incremental intensity from 75 to $90 \%$ of predicted maximal heart rate (Bishop, 2003). In the POST session the neuromuscular assessment started within $5-8 \mathrm{~min}$ after the race. A researcher was positioned at the finishing line to conduct the runners to the testing site, an indoor room located about $50 \mathrm{~m}$ from the finishing line. The testing session at POST lasted about $6 \mathrm{~min}$.

\section{Participants}

Twenty-three amateur male runners (age $45 \pm 9$ years, body mass $73 \pm 11 \mathrm{~kg}$, height $175 \pm 8 \mathrm{~cm}$ ) participated in this study. They were recruited through printed and electronic media, advertising the possibility to be a subject for scientific study within the event Run for Science (Lippi and Schena, 2017). Inclusion criteria were to be regularly engaged in recreational running (mean training regimen of $220 \mathrm{~min} /$ week), to have finished a halfmarathon in the previous 2 years, and to be free from clinical evidences of cardiovascular, neuromuscular, or joint diseases. All participants provided their written informed consent before participation in the experiments. The study was approved by the local Ethical Committee (Department of Neurological and Movement Sciences, University of Verona) and performed in accordance with the Helsinki Declaration.

\section{Data Acquisition}

For the assessment of knee extensor muscles, participants were seated on a custom-made chair with straps fastened across their chest and hips to avoid undesired lateral and frontal trunk displacements. During the testing, participants' knee and hip were flexed at $90^{\circ}$ from full extension and they were instructed to maintain the arms crossed on the chest. The knee extensors mechanical response was recorded with a strain gauge load cell (546QD- $220 \mathrm{~kg}$; DSEurope, Milan, Italy), fixed with noncompliant straps to the leg at the level of the external malleolus. All measurements were taken from the participants' right limb (which was the dominant limb for 21 out of 23 participants). Throughout the measurement session, visual feedback of the force output was provided as a real time signal displayed on a computer screen.

Electromyographic (EMG) signals were recorded from vastus medialis and vastus lateralis muscles with pairs of silver chloride $(\mathrm{Ag} / \mathrm{AgCl})$ circular (recording diameter of $10 \mathrm{~mm}$ ) surface electrodes (Kendall Meditrace 100) aligned to the muscle fibers according to guidelines (Rainoldi et al., 2004), with an interelectrode (center-to-center) distance of $20 \mathrm{~mm}$. The force and EMG signals were sampled at $2048 \mathrm{~Hz}$ and converted to digital data with a 12-bit A/D converter (EMG-USB, OT Bioelettronica, Turin, Italy). Force signal was conditioned with a moving averaging window of $0.01 \mathrm{~s}$ to remove noise. EMG signals were amplified with a gain of 500, band-pass filtered with a bandwidth frequency between 20 and $450 \mathrm{~Hz}$ (4th-order, zero lag Butterworth).

\section{Electrical Stimulation}

A constant current stimulator (Digitimer DS7A, Hertfordshire, United Kingdom) was used to deliver a square-wave stimulus of $1 \mathrm{~ms}$ duration with maximal voltage of $400 \mathrm{~V}$. The cathode $(2-\mathrm{cm}$ diameter, Meditrace 100 Kendall; Tyco, Markham, ON, Canada) and the anode $(5 \times 10 \mathrm{~cm}$; Compex, Ecublens, Switzerland $)$ were placed over the femoral nerve at the femoral triangle level beneath the inguinal ligament and on the lower part of the gluteal fold opposite to the cathode, respectively. Supra-maximal stimulation intensity level was determined by increasing the applied current until a plateau in maximal twitch was obtained. The stimulation intensity (range: $120-320 \mathrm{~mA}$ ) was then increased by $20 \%$, to ensure supra-maximal stimulus (120\% of optimal intensity) and kept constant throughout the experiment. The optimal placement for stimulation electrodes were marked on the skin during PRE to ensure the same position during POST.

\section{Procedure}

The neuromuscular test comprised two sets of the following: one maximal voluntary contraction (duration of $4 \mathrm{~s}$ ) of the knee extensors with super-imposed supra-maximal paired stimuli (doublet) at $100 \mathrm{~Hz}$ and followed (2 s intervals) by paired stimuli at $100 \mathrm{~Hz}$ (generating a high frequency doublet twitch, Db100), delivered in resting state. Then a paired stimulation at $10 \mathrm{~Hz}$ (generating a low frequency doublet twitch, Db10) and a single stimulation were delivered, interspaced by $5 \mathrm{~s}$, to evoke $\mathrm{M}$-wave and a resting twitch $(\mathrm{Tw})$. This was followed by $2 \mathrm{~min}$ of rest. 


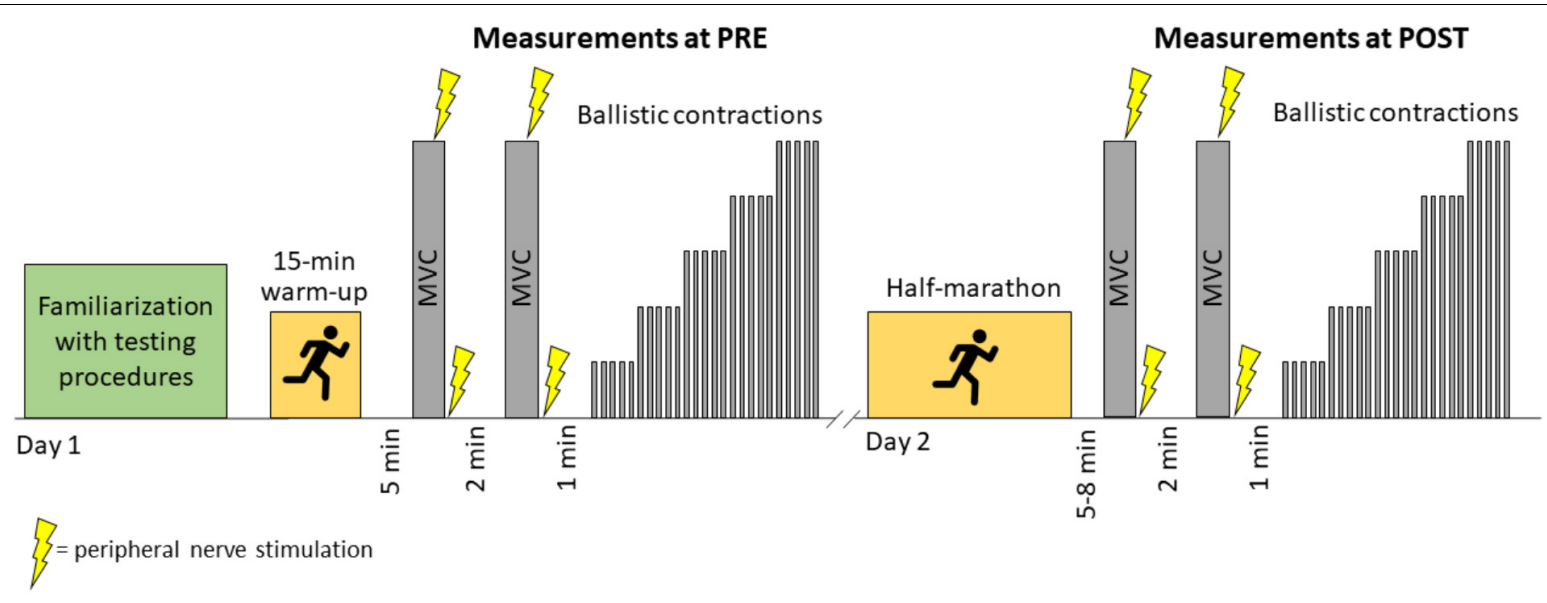

FIGURE 1 | Schematic representation of experimental procedures. MVC, maximal voluntary contraction.

If the difference between the two MVCF was higher than 5\%, a third set of this procedure was performed. Standardized verbal encouragements were provided to the participants during the execution of maximal voluntary contractions.

The RFD-SF protocol started 1 min after the last maximal voluntary contraction. Similar to the methods used by others (Freund and Budingen, 1978; Klass et al., 2008) the RFD-SF relationship was computed from sets of several fast and quick isometric contractions (ballistic contraction) performed across a full range of amplitudes (Figures 2A-C). Participants were instructed to produce isometric ballistic contractions in order to achieve peak force as quickly as possible and then relax instantly (Figure 2C). Participants performed five consecutive contractions at five different intensities presented in an ascending order $(20,40,60,80$, and $100 \%$ MVCF). The timing of contractions was cued by digital stimuli $4 \mathrm{~s}$ apart. Participants were explicitly instructed not to target the force levels requested because targeting slows the rate of force production (Gordon and Ghez, 1987). Instead, they were asked to produce fast contractions with peak forces reaching approximately the area around the target line. As previously suggested (Bellumori et al., 2011, 2013; Casartelli et al., 2014), in the familiarization session participants practiced until they felt comfortable with the task and could perform discrete ballistic contractions as instructed.

\section{Data Analysis \\ Mechanical Signals}

All data were analyzed by custom-written software in MATLAB R2015a (Mathworks, Natick, MA, United States). The amplitude of the resting doublet and single twitches (Db100, Db10, and $\mathrm{Tw}$ ) were analyzed and the average value computed from the two sets was considered. The level of voluntary activation (VA) during each maximal voluntary contraction was calculated as $\mathrm{VA}(\%)=100(1-$ interpolated doublet/Db100) $\times 100($ Merton, 1954). A correction was consistently applied to this equation when the superimposed doublet was elicited slightly before or after the actual peak force during a maximal voluntary contraction (Strojnik and Komi, 1998). The Db10:Db100 ratio was used as a surrogate measure of low-frequency fatigue, which is usually associated with a failure in the excitation-contraction coupling (Verges et al., 2009; Millet et al., 2011).

To calculate the RFD-SF, the force signal was firstly preprocessed using an overlapping moving window of $0.1 \mathrm{~s}$ (Bellumori et al., 2011). If a countermovement (i.e., a visible drop in force) was performed before the force onset, the contraction was discarded from the analysis. Then, the first derivative of the force signal was computed to obtain the RFD (N/s, see Figure 2B). For each subject and all contractions, peak force and peak RFD (which is local maximum of the RFD signal) were computed and plotted to obtain a linear regression (Figure 2D). Outliers were removed using the Cook distance methodology (Cook, 2000). The linear regression parameters (slope, y-intercept, $R^{2}$ ) were then calculated and considered as outcomes. In 14 occasions out of 46 (23 participants for two sessions, i.e., PRE and POST), the relationship between peak forces and peak RFD was not linear for the whole range of submaximal contractions. More precisely, it was linear from 0 to about $80-90 \%$ of the maximal force and then showed a logarithmic behavior from about $80-90 \%$ to the maximum. In these occasions, biphasic regression explained more variation than a linear regression between force and RFD (Linden, 2015). The breakpoint for this interrupted regression was calculated (Linden, 2015) and the coefficients for the first part of linear regression, i.e., up to the breakpoint, was reported. To compare the absolute values of RFD between subjects, the peak force and the peak RFD were then normalized to the MVCF (Richartz et al., 2010) obtained at PRE. Notably, the RFD data at POST were normalized with respect to the MVCF at PRE as well. This choice is important because we wanted to compare the RFD capacity between PRE vs. POST when targeting the same absolute torque, which was the first experimental question of this study. Normalizing the RFD for the MVCF at POST would have prevented to answer this question.

To assess if RFD exerted in rapid contractions of submaximal amplitude changed after the race, we compared the RFD 


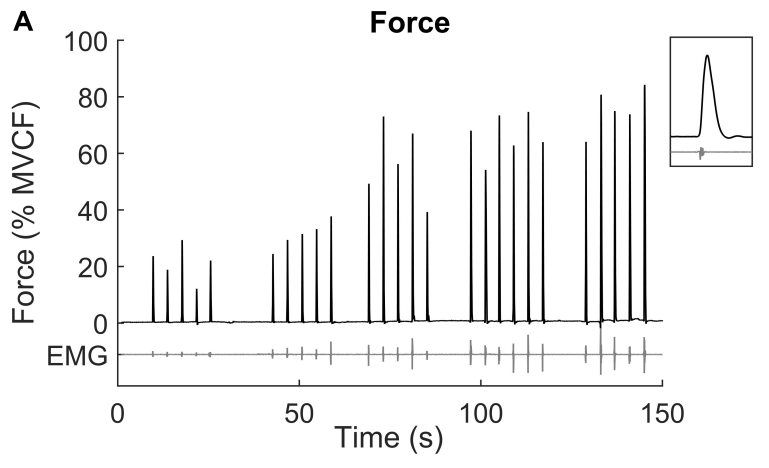

C

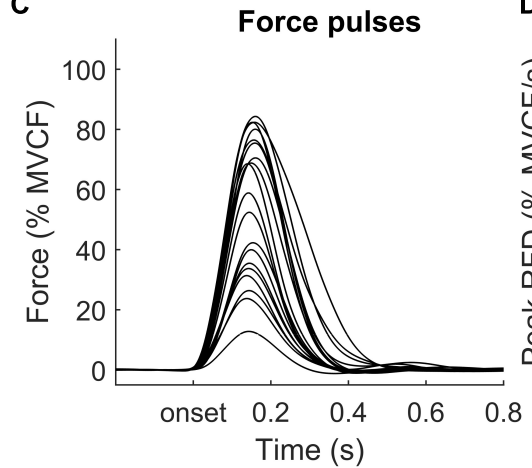

D

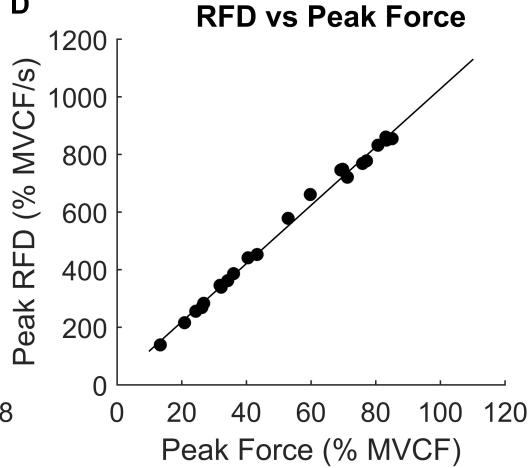

Rate of Force Development

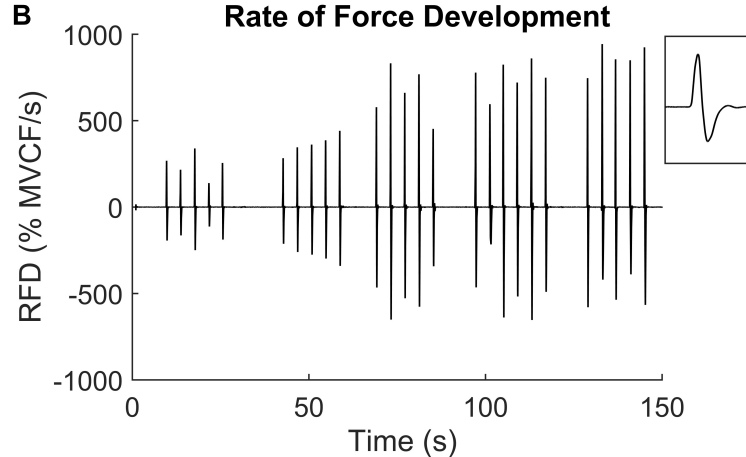

E

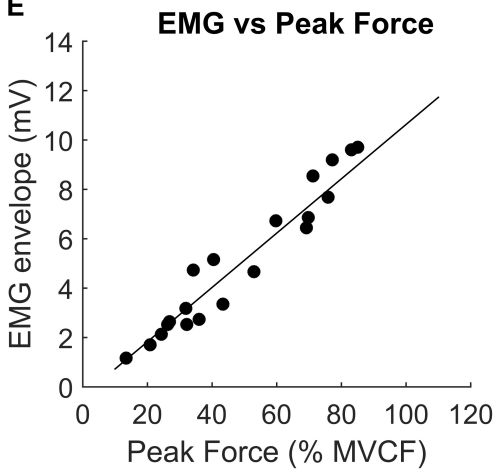

FIGURE 2 | Representative example of a set of ballistic contractions performed across a range of submaximal amplitudes. (A) Force signals recorded during 5 or 6 ballistic contraction for each force level; (B) RFD signals (first derivative of force); (C) superimposed force signals of each ballistic contraction; (D) each point represents the peak RFD ( $y$ value) and the peak force ( $x$ value) achieved in each contraction; (E) each point represents the electromyographic (EMG) envelope $(y$ value) and the peak force ( $x$ value) achieved in each contraction.

produced at the same absolute force level between PRE and POST. Since it is virtually impossible to have two ballistic contractions with the same amplitude in PRE and POST, we decided to evaluate the linear regression between RFD and force at predetermined force intervals across the whole available range of forces. To do this, the regression line of the RFD-SF was evaluated from $10 \%$ of the maximal force at PRE, to the highest available peak with $10 \%$ intervals (i.e., $10 \%, 20 \%, \ldots 100 \%$ of the MVCF at PRE).

Moreover, the maximal RFD was considered as the RFD recorded during the contraction that presented the highest RFD.

\section{EMG Signals}

M-wave properties, that is M-wave area ( $\left.\mathrm{M}_{\mathrm{AREA}}\right)$, peak-topeak amplitude ( $\mathrm{M}_{\text {AMPLITUDE}}$ ) and duration (M $\mathrm{M}_{\text {DURATION }}$ ) were measured from the EMG response obtained by single stimulations at rest. The root mean square (RMS) of EMG signals was calculated over a $500 \mathrm{~ms}$ epoch centered at the peak force of the maximal voluntary contraction with the greatest force $\left(\mathrm{RMS}_{\mathrm{MVC}}\right)$. The $\mathrm{RMS}_{\mathrm{MVC}} / \mathrm{M}_{\text {AMPLITUDE }}$ ratio was calculated as an index of muscle activation independently by changes in muscle excitability (Millet and Lepers, 2004). As recently suggested (Balshaw et al., 2017), the values coming from vastus medialis and vastus lateralis were averaged to increase within-participant reliability.
The EMG amplitude during RFD protocol was calculated as the envelope of the rectified EMG signal calculated in the 200-ms epoch before the RFD peak of each contraction (Figure 2E). The EMG value calculated as such may be considered as the overall amount of EMG activity that preceded the RFD peak. Since in this study the RFD peaks occurred at about 60-90 ms after the force onset, the length of the epoch ensured that the onset of EMG activity (which was not calculated) was included even when considering an electromechanical delay of $20-60 \mathrm{~ms}$. The EMG amplitude calculated as such was normalized to the $\mathrm{M}_{\text {AMPLITUDE }}$ to provide an index of muscle activation, independently by changes in muscle excitability.

\section{Statistical Analysis}

Statistical analysis was performed with MATLAB R2015a. Data are presented as mean \pm standard deviation (SD). KolmogorovSmirnov normality test was used to assess distributions normality. If the data were not normally distributed, they were log-transformed before statistical analysis and back-transformed to obtain descriptive statistics. Paired, two-tailed Student' $t$-tests were used to compare the RFD-SF linear regression parameters, mechanical, and EMG variables between PRE vs. POST. The level for statistical significance was set to $P<0.05$. Differences between PRE vs. POST were reported in absolute and percent values, the precision of estimates for absolute values was indicated with $90 \%$ confidence interval (CI). The magnitude of the difference was 
calculated as Cohen's $d$ effect size. Threshold values for effect size statistics were: $<0.2$, trivial; $>0.2$, small; $>0.6$, moderate; $>1.2$ large; $>2.0$, very large (Batterham and Hopkins, 2006).

\section{RESULTS}

Out of the 23 initially recruited participants, two individuals did not tolerate the peripheral nerve stimulation, thus data are reported for 21 participants. Their mean race time was $112 \pm 11 \mathrm{~min}$.

The results of the voluntary and evoked contractions are reported in Table 1. Briefly, MVCF and muscle activation during the maximal voluntary contraction $\left(\mathrm{RMS}_{\mathrm{MVC}}\right)$ decreased in POST with respect to PRE $(-21 \pm 10 \%$ and $-22 \pm 33 \%$, respectively). Furthermore, decreases in voluntary activation (VA: $-12 \pm 8 \%$ ), muscle contractile properties (Db100: $-12 \pm 8 \%$; Db10:Db100: $-9 \pm 10 \%)$, and muscle excitability (MAMPlitUdE: $-4 \pm 15 \%$; M AREA $: 7 \pm 13 \%$ ) were found. Maximal RFD and the associated EMG amplitude $\left(\mathrm{RMS}_{\mathrm{RFD}}\right)$ decreased after the race $(-19 \pm 18 \%$ and $-14 \pm 12 \%$, respectively).

The results of the RFD-SF are reported in Table 2. Briefly, all indices of the RFD-SF were unaffected by the race. Representative example of the relationship between RFD and force for PRE and POST is reported in Figure 3A. It can be seen that, in this specific participant, the RFD values at POST are substantially unchanged with respect to PRE up to the $60 \%$ of MVCF. This is confirmed by the evaluation of regression lines reported in the following paragraph.

The regression line was evaluated at PRE and POST from 10 to $70 \%$ of the MVCF at PRE. This was done because the decline in MVCF at POST made the force higher than $70 \%$ of MVCF unattainable for all participants. However, not every participant reached at POST the $70 \%$ of MVCF obtained at PRE. Thus, for 60 and $70 \%$ of MVCF the regression line was evaluated for only 12 and 6 participants, respectively. The RFD calculated across the available range (10-70\% of MVCF at PRE) did not change between PRE and POST (all $P$-values $>0.4$, Figure 4A). The EMG amplitude calculated in the same range did not change as well (see Figure 3B for a representative example and Figure 4B for group level results).

\section{DISCUSSION}

This study aimed to assess the effect of muscle fatigue induced by a half-marathon on the capacity to quickly contract leg muscles across a wide range of force levels production. The linear relationship existing between RFD and peak force, named RFD scaling factor, did not change as a consequence of fatigue. While the RFD exerted during short and fast contraction of maximal amplitudes decreased, the RFD exerted in short and fast contractions of submaximal amplitude was unaffected by fatigue. Evaluating the effects of fatigue on quick contractions of submaximal amplitudes has been mostly overlooked in the literature and it constitutes the innovation of this study. We advocate that this is relevant for endurance running and for many other sports and daily life activities which rely on quick contractions of submaximal amplitudes.

\section{Central and Peripheral Fatigue}

Shortly after the half-marathon, the maximal force decreased by $\approx 21 \%$, confirming that the prolonged run induced moderate muscle fatigue in knee extensors. This is in line with our previous studies where we also found a moderate decrease (ranged 11$24 \%$ ) in maximal force after half-marathons performed in similar experimental conditions (Boccia et al., 2017a,b, 2018). The strength loss is a common finding after endurance running in ecological race conditions: it increases non-linearly with the duration of exercises and it may vary between 8 and 41\% (Millet and Lepers, 2004; Place et al., 2010; Giandolini et al., 2016).

The decrease in maximal force was associated with a decrease of similar extent in EMG amplitude normalized to M-wave amplitude $\left(\mathrm{RMS}_{\mathrm{MVC}} / \mathrm{M}_{\mathrm{AMPLITUDE}} \approx 22 \%\right)$. While EMG amplitude per se cannot be used to measure the neural drive to the muscles (Martinez-Valdes et al., 2018), a decrement in the EMG amplitude normalized to the M-wave amplitude can be reasonably attributed to central mechanisms (Millet and Lepers, 2004). Furthermore, participants underwent a moderate decrease of voluntary activation $(\approx 12 \%)$. Together these findings indicated a reduced capacity to voluntarily activate the knee extensors in maximal contraction and reflect the presence of central fatigue. Central fatigue constitutes an important limitation to performance and is the major cause of the maximal force decrease induced by a prolonged whole body exercise such as running (Saldanha et al., 2008). Central fatigue could reflect the existence of a suboptimal neural drive output from the motor cortex (Taylor et al., 2006). It may nevertheless be located at spinal level (McNeil et al., 2011) and mediated by group IIIIV muscle afferents (Sidhu et al., 2017). The amount of central fatigue depends on many exercise parameters, such as intensity, duration, and contraction modality, among others (Taylor et al., 2016). While a detailed discussion of the mechanisms regulating the amount of central fatigue is beyond the scope of this paper, it is evident in the literature that central fatigue is overall greater for extreme-duration running (Millet and Lepers, 2004; Martin et al., 2010; Temesi et al., 2014) rather than for shorter, more intense bouts like half-marathons (Ross et al., 2010; Boccia et al., 2018).

In our investigation, there was also an evidence of peripheral fatigue, as indicated by a small decrease in the doublet amplitude of about $12 \%$. This result is in line with previous studies regarding a race of similar duration (Place et al., 2004; Boccia et al., 2018). The reduction in electrically evoked force can be attributed both to a decrease in sarcolemma membrane excitability (RodriguezFalces and Place, 2018), as evidenced by small decrease of $M$ wave amplitude $(\approx 4-7 \%)$, and to the impairment of the excitationcontraction coupling mechanism, as evidenced by the moderate decrement of Db10:Db100 ratio ( $\approx 9 \%$ ) (Place et al., 2010). In particular, the latter represents the disproportionate loss of force at low compared with high frequencies of electrical stimulation: it is termed low-frequency fatigue and it is most likely due to an impairment in calcium release or reuptake from the sarcoplasmic reticulum (Millet et al., 2011; Janecki et al., 2016). 
TABLE 1 | Voluntary and electrically evoked responses before and after the race.

\begin{tabular}{|c|c|c|c|c|c|c|c|c|c|c|c|}
\hline & \multicolumn{2}{|c|}{ PRE } & \multicolumn{2}{|c|}{ POST } & \multirow[t]{2}{*}{ Difference } & \multicolumn{2}{|c|}{$90 \% \mathrm{Cl}$} & \multicolumn{2}{|c|}{ Percent difference } & \multirow[t]{2}{*}{ Effect size (Cohen's d) } & \multirow[t]{2}{*}{$P$-value } \\
\hline & Mean & $S D$ & Mean & $S D$ & & Lower bound & Upper bound & Mean & $S D$ & & \\
\hline $\operatorname{MVCF}(N)$ & 524.8 & 144.7 & 426.3 & 159.3 & -98.5 & -110.6 & -86.4 & -20.6 & 9.6 & 0.64 & $<0.0001$ \\
\hline Maximal RFD (N/s) & 3747.7 & 801.2 & 3160.9 & 852.5 & -586.8 & -735.5 & -438.1 & -21.5 & 18.1 & 0.71 & $<0.0001$ \\
\hline VA (\%) & 91.1 & 7.2 & 83.3 & 10.2 & -7.7 & -9.6 & -5.8 & -8.7 & 6.5 & 0.81 & $<0.0001$ \\
\hline $\mathrm{RMS}_{\mathrm{MVC}} / \mathrm{M}_{\text {AMPLITUDE }}$ & 5.5 & 1.9 & 4.0 & 1.4 & -1.5 & -1.9 & -1.1 & -24.8 & 16.9 & 0.90 & $<0.0001$ \\
\hline $\mathrm{RMS}_{\mathrm{RFD}}(\mathrm{mV})$ & 1.82 & 0.45 & 1.48 & 0.41 & -0.29 & -0.46 & -0.12 & -27.2 & 45.8 & 0.79 & 0.0188 \\
\hline Db100 (N) & 250.5 & 43.7 & 226.4 & 47.8 & -24.2 & -31.6 & -16.8 & -9.8 & 8.8 & 0.53 & $<0.0001$ \\
\hline $\mathrm{RFD}_{\mathrm{Db} 100}(\mathrm{~N} / \mathrm{s})$ & 9244.4 & 2637.7 & 7580.8 & 2312.4 & -1663.5 & -2181.0 & -1146.1 & -16.8 & 16.4 & 0.67 & $<0.0001$ \\
\hline Db10 $(\mathrm{N})$ & 224.9 & 43.6 & 186.0 & 51.1 & -38.8 & -48.8 & -28.9 & -17.7 & 13.0 & 0.81 & $<0.0001$ \\
\hline Db10:Db100 (\%) & 89.8 & 9.2 & 81.8 & 12.8 & -7.9 & -10.8 & -5.1 & -9.0 & 9.2 & 0.63 & 0.0004 \\
\hline Single twitch (N) & 157.1 & 34.1 & 133.5 & 36.4 & -23.7 & -31.0 & -16.4 & -15.1 & 13.5 & 0.69 & $<0.0001$ \\
\hline MDURATION (ms) & 20.9 & 1.1 & 20.9 & 1.2 & 0.0 & -0.3 & 0.3 & 0.2 & 4.5 & 0.00 & 0.8501 \\
\hline $\mathrm{M}_{\text {AMPLITUDE }}(\mathrm{mV})$ & 2.70 & 0.63 & 2.52 & 0.54 & -0.17 & -0.29 & -0.06 & -4.7 & 14.6 & 0.31 & 0.0266 \\
\hline $\mathrm{M}_{\text {AREA }}(\mathrm{mV} \cdot \mathrm{ms})$ & 0.374 & 0.091 & 0.346 & 0.075 & -0.028 & -0.043 & -0.012 & -5.9 & 12.6 & 0.33 & 0.0112 \\
\hline
\end{tabular}

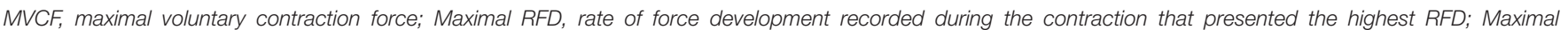

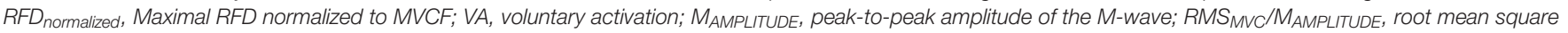

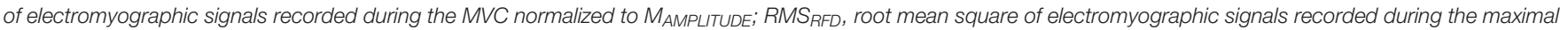

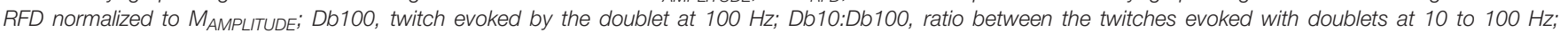
MDURATION, duration of the M-wave; MAREA, total area of the M-wave.

TABLE 2 | Parameters of the linear regression between force and rate of force development achieved during the ballistic contractions before and after the race.

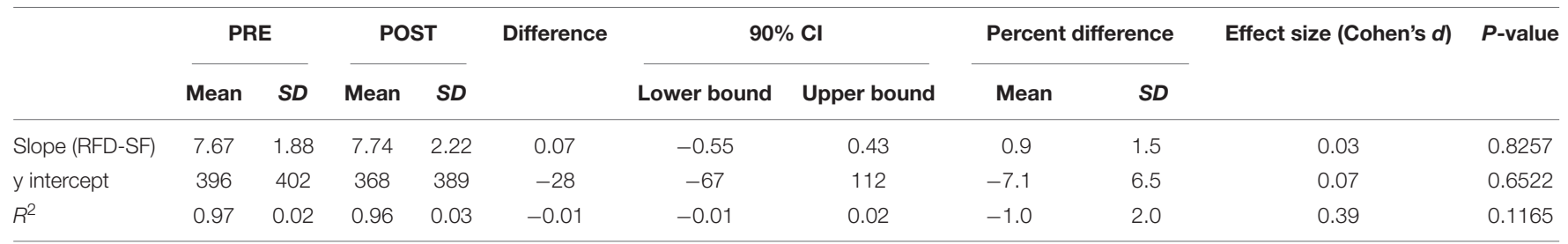

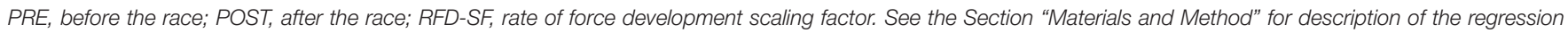
line.
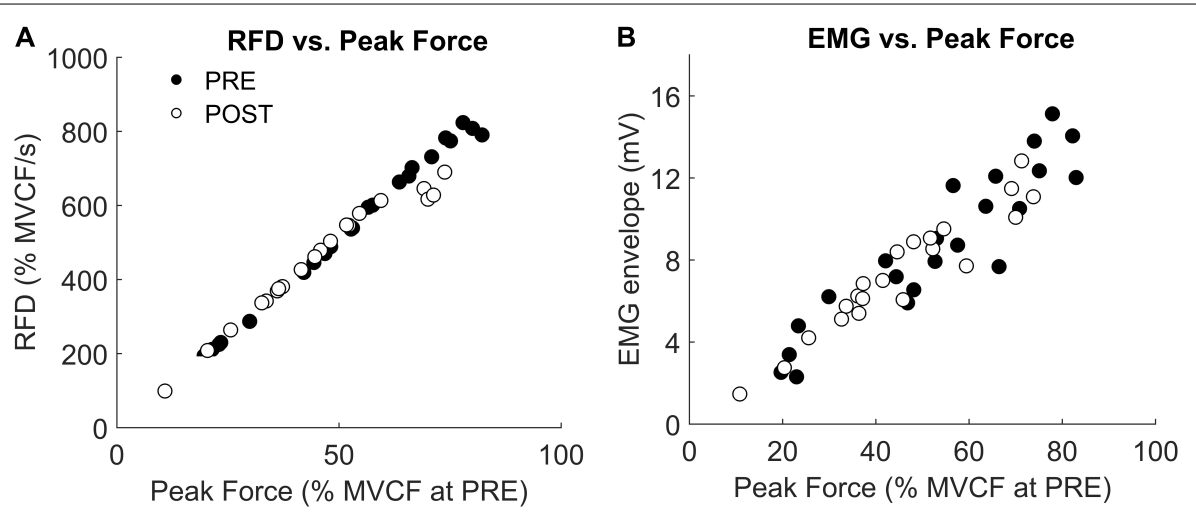

FIGURE 3 | Representative example of (A) rate of force development (RFD) and (B) electromyographic (EMG) envelope recorded before (PRE) and after (POST) half marathon in a set of ballistic contraction performed across a range of submaximal amplitudes. Force data are normalized with respect to the maximal voluntary contraction force (MVCF) recorded at PRE.

For sure, the delay ( $5-8 \mathrm{~min}$ ) between the end of the race and the beginning of the testing allowed a partial recovery in both central (Gruet et al., 2014; Mira et al., 2017) and peripheral fatigue (Froyd et al., 2013). Indeed, it has been shown that substantial central and peripheral recovery occurs in the first $5 \mathrm{~min}$ after task completion (Carroll et al., 2017). Thus, during final part of the race, that is when fatigue possibly influenced mainly the running performance, the central and peripheral fatigue were likely greater than the ones recorded in the measurements. Therefore, the impairment that we found in maximal force 

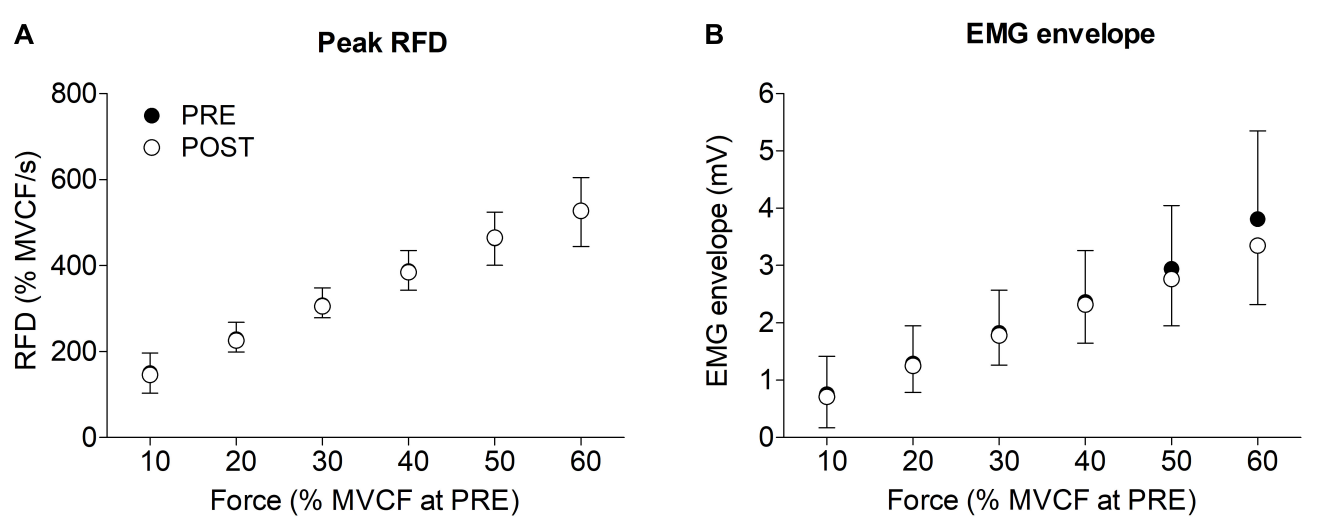

FIGURE 4 | Mean and standard deviation of (A) rate of force development (RFD) and (B) electromyographic (EMG) envelope before (PRE) and after (POST) half marathon. The values are reported from 10 to $60 \%$ of maximal voluntary contraction force (MVCF) recorded before the race (see methods).

and RFD (discussed below) were likely underestimated as well. However, since the measurements of central and peripheral fatigue were concomitant (within few seconds) to the maximal force and RFD assessment, the magnitude of fatigue reported here is the level of fatigue that influenced the maximal force and RFD measurements.

\section{RFD in Contractions of Maximal and Submaximal Amplitude}

The maximal RFD moderately decreased after the race $(\approx 19 \%)$. This is in line with some previous studies investigating prolonged running (Kelly et al., 2011; Oliveira et al., 2013; Boccia et al., 2017a, 2018). The muscle activation (EMG amplitude normalized to M-wave amplitude) measured during the execution of maximal RFD moderately decreased $(\approx 14 \%)$, suggesting that the impairment in RFD can be attributed, at least in part, to a suboptimal neural drive to the muscles (Maffiuletti et al., 2016). However, the observed peripheral fatigue may have influenced the maximal RFD as well (Maffiuletti et al., 2016).

The most interesting result of this study was that despite the presence of significant amount of central and peripheral fatigue, the capacity to quickly produce ballistic contraction of submaximal amplitude remained unaffected. Indeed, the RFD recorded during submaximal tasks, was maintained after the half-marathon. Because of the drop in maximal force, after the race the participants were not able to reach force levels higher than $70 \%$ of the maximal force produced before the race. However, up to that level, the RFD values recorded in fatigued state were similar to those recorded in fresh state. Interestingly, a previous study (Kulmala et al., 2016) found that the peak force exerted by the knee extensors when running at $4.1 \mathrm{~m} \cdot \mathrm{s}^{-1}$ reached the $\approx 63 \%$ of maximal force, which is below the limit where the RFD was unaltered by fatigue in the present study. Together, these findings can explain why a recent meta-analysis showed that muscle fatigue did not significantly change the ground reaction force active peak and loading rate in running (Zadpoor and Nikooyan, 2012). To understand if this non-different RFD was associated to changes in muscle activation, the EMG amplitude, normalized to the M-wave amplitude, was calculated from the beginning of the contraction to the peak RFD of each ballistic contraction. An increase in EMG amplitude would mean that the central nervous system should increase muscles activation to produce the same RFD, thus suggesting a decrease in neuromuscular efficiency. However, the EMG amplitude did not change after the race (Figures 3B, 4B) suggesting that to produce RFD in contractions of submaximal amplitudes the central nervous system does not have to increase the neural drive to the muscles. Therefore, the RFD in fatigued state was produced as effectively as in fresh state for a wide range of submaximal contractions. Runners are accustomed to repeatedly cope with the transient of the vertical ground reaction force within the first 100-150 ms of stance (Lieberman et al., 2010). Thus, this finding may be attributed to the specific population of habitual runners recruited in this study. Further studies are needed to understand if different population and/or fatiguing exercises would give different results.

The fact that only the maximal RFD was affected by fatigue poses interesting questions about the effects of fatigue on muscle performance. As the RFD recorded during maximal contractions is higher than the RFD calculated during submaximal contractions it is possible to hypothesize that fatigue affected the RFD only when the increase of force was at its highest possible rate. When performing maximal explosive contractions high-threshold motor units are recruited since the beginning of the contraction, at very low force level (Heckman and Enoka, 2012). However, this is not true when the contraction is slower and the transient of force is less steep (Heckman and Enoka, 2012). Together, this finding may allow to speculate that central fatigue results in inability to activate high-threshold motor units, while preserving the capacity to activate low threshold motor units, thus maintaining the capacity to produce less steep RFD. The same can be said for peripheral fatigue: while the lowered contractile properties may reduce the capacity to produce maximal RFD (Jones, 2010), the residual contractile properties seemed to be enough to produce less steep RFD. While we still agree on the goodness of measuring the maximal RFD as an index of muscle fatigue, the 
decrease of maximal RFD unlikely has a direct effect on mechanics of long duration exercises. In an attempt to measure neuromuscular adjustments that mechanistically modulate performance (Enoka and Duchateau, 2016), we propose that the measurement of RFD on quick contractions of submaximal amplitude may be more relevant than maximal RFD in endurance context.

\section{RFD-SF}

Since RFD-SF was generally associated with features of muscle contraction quickness, we hypothesized that this parameter should be altered by fatigue, but this was not the case (Figure 3A and Table 2). As it can be seen from the data of a representative participant reported in Figure $\mathbf{3 A}$, the linear regressions before and after the running were closely similar up the $60-70 \%$ of maximal force recorded before the race. Fatigue lowered the maximal available force, consequently limiting the extent to which the regression extends rightward but did not change the slope of the regression. This result, obtained with an original experimental approach, confirmed that the RFD-SF is independent from muscle strength (Bellumori et al., 2011; Casartelli et al., 2014; Djordjevic and Uygur, 2017). Furthermore, it demonstrated for the first time that RFD-SF is independent from the fatigue induced by a prolonged running race. Even though maximal muscle strength decreased by $21 \%$ after the race, the RFD-SF remained unaltered. Furthermore, this result helps to shed light on the neuromuscular correlates of RFD-SF, by excluding moderate muscle fatigue from the potential determinants. Since we observed a moderate decrease in voluntary activation and muscle contractile properties, this result suggests that a moderate impairment in both central and peripheral neuromuscular properties did not affect the RFD-SF. However, future studies should investigate if different exercise modality and/or more severe muscle fatigue may affect the RFD-SF.

While the slope of the regression between force and RFD is usually adopted as the main parameter, the intercept of this regression may also be of interest. Indeed, fatigue could potentially shift downward the regression, without modifying its slope. Contrary to the expectation, the intercept of the linear regression was unaffected by fatigue as well (Table 2). Together, these results suggest that (at least across submaximal forces) the relationship between force and RFD remained unaltered in fatigued condition. Hence the RFD-SF cannot be used as an index of fatigue on the basis of the herein findings. However, since it can provide a measure of contraction quickness independently from the decrease in maximal force that can be expected in presence of fatigue, potential usefulness of RFD-SF in the context of muscle fatigue is still to be determined.

\section{Limitations}

The results of this study were specific to the type of exercise adopted to induce fatigue, i.e., prolonged running of about $2 \mathrm{~h}$. It is not possible to infer how the RFD and RFD-SF would behave when adopting different fatiguing protocols. Furthermore, the participants were habitual recreational runners, thus our results cannot be generalized to untrained population.
While the EMG activity during the ballistic contractions was unaffected by fatigue, it is reasonable to think that the recording of mechanomyogram would have provided insightful information about the mechanical determinants of this type of contraction. Indeed, mechanomyograms features were reported to be sensitive to fatigue even when the EMG response was unaltered (Perry-Rana et al., 2002) and were useful to identify possible mechanical adjustments occurring across a series of brief isometric contraction from 10 to $100 \%$ of maximal force (Beck et al., 2004). For these reasons, future studies are warranted to include mechanomyogram analysis to further elucidate the effect of fatigue on this type of contractions.

Another main limitation was that while we measured the performance fatigability (i.e., the mechanical output) of the participants, we did not measure the perceived fatigability, see (Enoka and Duchateau, 2016) for review. Indeed, while we demonstrated that to produce ballistic contraction of submaximal amplitudes the RFD and the associated muscle activation were the same after the race, this does not mean that participants perceived the same effort to produce the ballistic contractions. Thus, future studies are warranted to understand if this preservation of RFD in ballistic contractions would be associated to possibly higher perceived effort.

\section{CONCLUSION AND PERSPECTIVES}

To summarize, long-distance running of about $2 \mathrm{~h}$ affects the capacity to quickly produce force during ballistic contractions of maximal, but not submaximal, amplitudes. The RFD scaling factor was also unaffected after the race. Overall, these findings suggest that central and peripheral fatigue did not affect the quickness of contraction across a wide range of submaximal force levels. This is a relevant finding because running, as many other endurance sport and daily life activities, relies on fast and brief contractions aimed to produce submaximal, rather than maximal, force levels. Future studies should assess if this capacity may be affected by fatiguing task of different intensity, length and contraction modality.

\section{AUTHOR CONTRIBUTIONS}

GB and DD: conceptualization. GB, DD, PRB, CZ, CT, LF, and BP: methodology. GB, DD, PRB, CZ, CT, LF, BP, FS, and AR: investigation. $\mathrm{GB}$ and $\mathrm{PRB}$ : formal analysis. $\mathrm{GB}$ : writing - original draft. GB, DD, PRB, CZ, CT, LF, BP, FS, and AR: writing - review and editing. FS and AR: supervision.

\section{ACKNOWLEDGMENTS}

The authors wish to thank all the athletes who participated to the study. We also thank Lorenzo Bortolan for his valuable help with technical issues and Antonio La Torre for his contribution in the planning and development of this research project. 


\section{REFERENCES}

Balshaw, T. G., Fry, A., Maden-Wilkinson, T. M., Kong, P. W., and Folland, J. P. (2017). Reliability of quadriceps surface electromyography measurements is improved by two vs. single site recordings. Eur. J. Appl. Physiol. 117, 1085-1094. doi: 10.1007/s00421-017-3595-z doi: 10.1007/s00421-017-3595-z

Batterham, A. M., and Hopkins, W. G. (2006). Making meaningful inferences about magnitudes. Int. J. Sports Physiol. Perform. 1, 50-57. doi: 10.1123/ijspp.1.1.50

Beck, T. W., Housh, T. J., Johnson, G. O., Weir, J. P., Cramer, J. T., Coburn, J. W., et al. (2004). Mechanomyographic amplitude and mean power frequency versus torque relationships during isokinetic and isometric muscle actions of the biceps brachii. J. Electromyogr. Kinesiol. 14, 555-564. doi: 10.1016/j.jelekin. 2004.03.001

Bellumori, M., Jaric, S., and Knight, C. A. (2011). The rate of force development scaling factor (RFD-SF): protocol, reliability, and muscle comparisons. Exp. Brain Res. 212, 359-369. doi: 10.1007/s00221-011-2735-7

Bellumori, M., Jaric, S., and Knight, C. A. (2013). Age-related decline in the rate of force development scaling factor. Motor Control 17, 370-381. doi: 10.1123/mcj. 17.4.370

Bellumori, M., Uygur, M., and Knight, C. A. (2017). High-speed cycling intervention improves rate-dependent mobility in older adults. Med. Sci. Sports Exerc. 49, 106-114. doi: 10.1249/MSS.0000000000001069

Bigouette, J., Simon, J., Liu, K., and Docherty, C. L. (2016). Altered vertical ground reaction forces in participants with chronic ankle instability while running. J. Athl. Train. 51, 682-687. doi: 10.4085/1062-6050-51.11.11

Bishop, D. (2003). Warm up I: potential mechanisms and the effects of passive warm up on exercise performance. Sports Med. 33, 439-454. doi: 10.2165/ 00007256-200333060-00005

Boccia, G., Dardanello, D., Tarperi, C., Festa, L., La Torre, A., Pellegrini, B., et al. (2017a). Fatigue-induced dissociation between rate of force development and maximal force across repeated rapid contractions. Hum. Mov. Sci. 54, 267-275. doi: 10.1016/j.humov.2017.05.016

Boccia, G., Dardanello, D., Tarperi, C., Rosso, V., Festa, L., La Torre, A., et al. (2017b). Decrease of muscle fiber conduction velocity correlates with strength loss after an endurance run. Physiol. Meas. 38, 233-240. doi: 10.1088/1361$6579 /$ aa5139

Boccia, G., Dardanello, D., Tarperi, C., Festa, L., La Torre, A., Pellegrini, B., et al. (2018). Women show similar central and peripheral fatigue to men after halfmarathon. Eur. J. Sport Sci. 18, 695-704. doi: 10.1080/17461391.2018.1442500

Boccia, G., Dardanello, D., Zoppirolli, C., Bortolan, L., Cescon, C., Schneebeli, A., et al. (2016). Central and peripheral fatigue in knee and elbow extensor muscles after a long-distance cross-country ski race. Scand. J. Med. Sci. Sports 27, 945-955. doi: 10.1111/sms.12718

Buckthorpe, M., Pain, M. T., and Folland, J. P. (2014). Central fatigue contributes to the greater reductions in explosive than maximal strength with high-intensity fatigue. Exp. Physiol. 99, 964-973. doi: 10.1113/expphysiol.2013.075614

Carroll, T. J., Taylor, J. L., and Gandevia, S. C. (2017). Recovery of central and peripheral neuromuscular fatigue after exercise. J. Appl. Physiol. 122, 10681076. doi: 10.1152/japplphysiol.00775.2016

Casartelli, N. C., Lepers, R., and Maffiuletti, N. A. (2014). Assessment of the rate of force development scaling factor for the hip muscles. Muscle Nerve 50, 932-938. doi: $10.1002 /$ mus. 24229

Cook, R. D. (2000). Detection of influential observation in linear regression. Technometrics 42, 65-68. doi: 10.1080/00401706.2000.10485981

Djordjevic, D., and Uygur, M. (2017). Methodological considerations in the calculation of the rate of force development scaling factor. Physiol. Meas. 39:015001. doi: 10.1088/1361-6579/aa9f51

Enoka, R. M., and Duchateau, J. (2016). Translating fatigue to human performance. Med. Sci. Sports Exerc. 48, 2228-2238. doi: 10.1249/MSS.0000000000000929

Folland, J. P., Buckthorpe, M. W., and Hannah, R. (2014). Human capacity for explosive force production: neural and contractile determinants. Scand. J. Med. Sci. Sports 24, 894-906. doi: 10.1111/sms.12131

Freund, H. J., and Budingen, H. J. (1978). The relationship between speed and amplitude of the fastest voluntary contractions of human arm muscles. Exp. Brain Res. 31, 1-12. doi: 10.1007/BF00235800

Froyd, C., Millet, G. Y., and Noakes, T. D. (2013). The development of peripheral fatigue and short-term recovery during self-paced high-intensity exercise. J. Physiol. 591, 1339-1346. doi: 10.1113/jphysiol.2012.245316
Gandevia, S. C. (2001). Spinal and supraspinal factors in human muscle fatigue. Physiol. Rev. 81, 1725-1789. doi: 10.1152/physrev.2001.81.4.1725

Ghez, C., and Vicario, D. (1978). The control of rapid limb movement in the cat. II. Scaling of isometric force adjustments. Exp. Brain Res. 33, 191-202. doi: 10.1007/BF00238059

Giandolini, M., Vernillo, G., Samozino, P., Horvais, N., Edwards, W. B., Morin, J. B., et al. (2016). Fatigue associated with prolonged graded running. Eur. J. Appl. Physiol. 116, 1859-1873. doi: 10.1007/s00421-016-3437-4

Girard, O., Nybo, L., Mohr, M., and Racinais, S. (2015). Plantar flexor neuromuscular adjustments following match-play football in hot and cool conditions. Scand. J. Med. Sci. Sports 25(Suppl. 1), 154-163. doi: 10.1111/sms. 12371

Gordon, J., and Ghez, C. (1987). Trajectory control in targeted force impulses. II. Pulse height control. Exp. Brain Res. 67, 241-252. doi: 10.1007/BF0024 8546

Gruet, M., Temesi, J., Rupp, T., Levy, P., Verges, S., and Millet, G. Y. (2014). Dynamics of corticospinal changes during and after high-intensity quadriceps exercise. Exp. Physiol. 99, 1053-1064. doi: 10.1113/expphysiol.2014.078840

Heckman, C. J., and Enoka, R. M. (2012). Motor unit. Compr. Physiol. 2, 26292682. doi: 10.1002/cphy.c100087

Janecki, D., Jaskolska, A., Marusiak, J., and Jaskolski, A. (2016). Low-frequency fatigue assessed as double to single twitch ratio after two bouts of eccentric exercise of the elbow flexors. J. Sports Sci. Med. 15, 697-703.

Jones, D. A. (2010). Changes in the force-velocity relationship of fatigued muscle: implications for power production and possible causes. J. Physiol. 588(Pt 16), 2977-2986. doi: 10.1113/jphysiol.2010.190934

Kelly, L. A., Girard, O., and Racinais, S. (2011). Effect of orthoses on changes in neuromuscular control and aerobic cost of a 1-h run. Med. Sci. Sports Exerc. 43, 2335-2343. doi: 10.1249/MSS.0b013e31822037ca

Klass, M., Baudry, S., and Duchateau, J. (2008). Age-related decline in rate of torque development is accompanied by lower maximal motor unit discharge frequency during fast contractions. J. Appl. Physiol. 104, 739-746. doi: 10.1152/ japplphysiol.00550.2007

Kluitenberg, B., Bredeweg, S. W., Zijlstra, S., Zijlstra, W., and Buist, I. (2012). Comparison of vertical ground reaction forces during overground and treadmill running. A validation study. BMC Musculoskelet. Disord. 13:235. doi: 10.1186/ 1471-2474-13-235

Kulmala, J. P., Korhonen, M. T., Ruggiero, L., Kuitunen, S., Suominen, H., Heinonen, A., et al. (2016). Walking and running require greater effort from the ankle than the knee extensor muscles. Med. Sci. Sports Exerc. 48, 2181-2189. doi: 10.1249/MSS.0000000000001020

Kyrolainen, H., Avela, J., and Komi, P. V. (2005). Changes in muscle activity with increasing running speed. J. Sports Sci. 23, 1101-1109. doi: 10.1080/ 02640410400021575

Lieberman, D. E., Venkadesan, M., Werbel, W. A., Daoud, A. I., D’Andrea, S., Davis, I. S., et al. (2010). Foot strike patterns and collision forces in habitually barefoot versus shod runners. Nature 463, 531-535. doi: 10.1038/nature0 8723

Linden, A. (2015). Conducting interrupted time-series analysis for single- and multiple-group comparisons. Stata J. 15, 480-500.

Lippi, G., and Schena, F. (2017). Run for Science (R4S): the history of a successful project of precision and laboratory medicine in sport and exercise. J. Lab. Precis. Med. 2:11. doi: 10.21037/jlpm.2017.04.01

Maffiuletti, N. A., Aagaard, P., Blazevich, A. J., Folland, J., Tillin, N., and Duchateau, J. (2016). Rate of force development: physiological and methodological considerations. Eur. J. Appl. Physiol. 116, 1091-1116. doi: 10. 1007/s00421-016-3346-6

Martin, V., Kerherve, H., Messonnier, L. A., Banfi, J. C., Geyssant, A., Bonnefoy, R., et al. (2010). Central and peripheral contributions to neuromuscular fatigue induced by a 24-h treadmill run. J. Appl. Physiol. 108, 1224-1233. doi: 10.1152/ japplphysiol.01202.2009

Martinez-Valdes, E., Negro, F., Falla, D., De Nunzio, A. M., and Farina, D. (2018) Surface electromyographic amplitude does not identify differences in neural drive to synergistic muscles. J. Appl. Physiol. 124, 1071-1079. doi: 10.1152/ japplphysiol.01115.2017

McLellan, C. P., Lovell, D. I., and Gass, G. C. (2011). The role of rate of force development on vertical jump performance. J. Strength Cond. Res. 25, 379-385. doi: 10.1519/JSC.0b013e3181be305c 
McNeil, C. J., Giesebrecht, S., Gandevia, S. C., and Taylor, J. L. (2011). Behaviour of the motoneurone pool in a fatiguing submaximal contraction. J. Physiol. 589(Pt 14), 3533-3544. doi: 10.1113/jphysiol.2011.207191

Merton, P. A. (1954). Voluntary strength and fatigue. J. Physiol. 123, 553-564. doi: 10.1113/jphysiol.1954.sp005070

Millet, G. Y., and Lepers, R. (2004). Alterations of neuromuscular function after prolonged running, cycling and skiing exercises. Sports Med. 34, 105-116. doi: 10.2165/00007256-200434020-00004

Millet, G. Y., Martin, V., Martin, A., and Verges, S. (2011). Electrical stimulation for testing neuromuscular function: from sport to pathology. Eur. J. Appl. Physiol. 111, 2489-2500. doi: 10.1007/s00421-011-1996-y

Mira, J., Lapole, T., Souron, R., Messonnier, L., Millet, G. Y., and Rupp, T. (2017). Cortical voluntary activation testing methodology impacts central fatigue. Eur. J. Appl. Physiol. 117, 1845-1857. doi: 10.1007/s00421-017-3678-x

Morel, B., Rouffet, D. M., Saboul, D., Rota, S., Clemencon, M., and Hautier, C. A. (2015). Peak torque and rate of torque development influence on repeated maximal exercise performance: contractile and neural contributions. PLoS One 10:e0119719. doi: 10.1371/journal.pone.0119719

Oliveira, A. S., Caputo, F., Aagaard, P., Corvino, R. B., Goncalves, M., and Denadai, B. S. (2013). Isokinetic eccentric resistance training prevents loss in mechanical muscle function after running. Eur. J. Appl. Physiol. 113, 2301-2311. doi: 10.1007/s00421-013-2660-2665

Perry-Rana, S. R., Housh, T. J., Johnson, G. O., Bull, A. J., Berning, J. M., and Cramer, J. T. (2002). MMG and EMG responses during fatiguing isokinetic muscle contractions at different velocities. Muscle Nerve 26, 367-373. doi: $10.1002 /$ mus. 10214

Place, N., Lepers, R., Deley, G., and Millet, G. Y. (2004). Time course of neuromuscular alterations during a prolonged running exercise. Med. Sci. Sports Exerc. 36, 1347-1356. doi: 10.1249/01.MSS.0000135786.22996.77

Place, N., Yamada, T., Bruton, J. D., and Westerblad, H. (2010). Muscle fatigue: from observations in humans to underlying mechanisms studied in intact single muscle fibres. Eur. J. Appl. Physiol. 110, 1-15. doi: 10.1007/s00421-010-14801480

Rainoldi, A., Melchiorri, G., and Caruso, I. (2004). A method for positioning electrodes during surface EMG recordings in lower limb muscles. J. Neurosci. Methods 134, 37-43. doi: 10.1016/j.jneumeth.2003.10.014

Richartz, C., Levenez, M., Boucart, J., and Duchateau, J. (2010). Initial conditions influence the characteristics of ballistic contractions in the ankle dorsiflexors. Eur. J. Appl. Physiol. 110, 805-814. doi: 10.1007/s00421-010-1564-x

Rodriguez-Falces, J., and Place, N. (2018). Determinants, analysis and interpretation of the muscle compound action potential ( $M$ wave) in humans: implications for the study of muscle fatigue. Eur. J. Appl. Physiol. 118, 501-521. doi: 10.1007/s00421-017-3788-5

Rodriguez-Rosell, D., Pareja-Blanco, F., Aagaard, P., and Gonzalez-Badillo, J. J. (2017). Physiological and methodological aspects of rate of force development assessment in human skeletal muscle. Clin. Physiol. Funct. Imaging 38, 743-762. doi: $10.1111 /$ cpf.12495

Ross, E. Z., Goodall, S., Stevens, A., and Harris, I. (2010). Time course of neuromuscular changes during running in well-trained subjects. Med. Sci. Sports Exerc. 42, 1184-1190. doi: 10.1249/MSS.0b013e3181c91f4e

Saldanha, A., Nordlund Ekblom, M. M., and Thorstensson, A. (2008). Central fatigue affects plantar flexor strength after prolonged running. Scand. J. Med. Sci. Sports 18, 383-388. doi: 10.1111/j.1600-0838.2007.00721.x

Sidhu, S. K., Weavil, J. C., Mangum, T. S., Jessop, J. E., Richardson, R. S., Morgan, D. E., et al. (2017). Group III/IV locomotor muscle afferents alter motor cortical and corticospinal excitability and promote central fatigue during cycling exercise. Clin. Neurophysiol. 128, 44-55. doi: 10.1016/j.clinph.2016.10. 008

Strojnik, V., and Komi, P. V. (1998). Neuromuscular fatigue after maximal stretchshortening cycle exercise. J. Appl. Physiol. 84, 344-350. doi: 10.1152/jappl.1998. 84.1.344

Taylor, J. L., Amann, M., Duchateau, J., Meeusen, R., and Rice, C. L. (2016). Neural contributions to muscle fatigue: from the brain to the muscle and back again. Med. Sci. Sports Exerc. 48, 2294-2306. doi: 10.1249/Mss.000000000000 0923

Taylor, J. L., Todd, G., and Gandevia, S. C. (2006). Evidence for a supraspinal contribution to human muscle fatigue. Clin. Exp. Pharmacol. Physiol. 33, 400-405. doi: 10.1111/j.1440-1681.2006.04363.x

Temesi, J., Rupp, T., Martin, V., Arnal, P. J., Feasson, L., Verges, S., et al. (2014). Central fatigue assessed by transcranial magnetic stimulation in ultratrail running. Med. Sci. Sports Exerc. 46, 1166-1175. doi: 10.1249/MSS. 0000000000000207

Van Cutsem, M., and Duchateau, J. (2005). Preceding muscle activity influences motor unit discharge and rate of torque development during ballistic contractions in humans. J. Physiol. 562(Pt 2), 635-644. doi: 10.1113/jphysiol. 2004.074567

Van Cutsem, M., Duchateau, J., and Hainaut, K. (1998). Changes in single motor unit behaviour contribute to the increase in contraction speed after dynamic training in humans. J. Physiol. 513( Pt 1), 295-305. doi: 10.1111/j.1469-7793. 1998.295by.x

Verges, S., Maffiuletti, N. A., Kerherve, H., Decorte, N., Wuyam, B., and Millet, G. Y. (2009). Comparison of electrical and magnetic stimulations to assess quadriceps muscle function. J. Appl. Physiol. 106, 701-710. doi: 10.1152/ japplphysiol.01051.2007

Wierzbicka, M. M., Wiegner, A. W., Logigian, E. L., and Young, R. R. (1991). Abnormal most-rapid isometric contractions in patients with Parkinson's disease. J. Neurol. Neurosurg. Psychiatry 54, 210-216. doi: 10.1136/jnnp.5 4.3.21

Wilson, G. J., and Murphy, A. J. (1996). The use of isometric tests of muscular function in athletic assessment. Sports Med. 22, 19-37. doi: 10.2165/00007256199622010-00003

Zadpoor, A. A., and Nikooyan, A. A. (2012). The effects of lower extremity muscle fatigue on the vertical ground reaction force: a meta-analysis. Proc. Inst. Mech. Eng. H 226, 579-588. doi: 10.1177/0954411912447021

Zaras, N. D., Stasinaki, A. N., Methenitis, S. K., Krase, A. A., Karampatsos, G. P., Georgiadis, G. V., et al. (2016). Rate of force development, muscle architecture, and performance in young competitive track and field throwers. J. Strength Cond. Res. 30, 81-92. doi: 10.1519/JSC.0000000000001048

Conflict of Interest Statement: The authors declare that the research was conducted in the absence of any commercial or financial relationships that could be construed as a potential conflict of interest.

Copyright (c) 2018 Boccia, Dardanello, Brustio, Tarperi, Festa, Zoppirolli, Pellegrini, Schena and Rainoldi. This is an open-access article distributed under the terms of the Creative Commons Attribution License (CC BY). The use, distribution or reproduction in other forums is permitted, provided the original author(s) and the copyright owner(s) are credited and that the original publication in this journal is cited, in accordance with accepted academic practice. No use, distribution or reproduction is permitted which does not comply with these terms. 